\title{
Features of the popliteal lymph nodes seen on musculoskeletal MRI in a Western population
}

\author{
Gijsbert Daniel Musters • Roeland P. Kleipool • \\ Shandra Bipat • Mario Maas
}

Received: 4 October 2010 /Revised: 15 December 2010 /Accepted: 29 December 2010 /Published online: 20 January 2011

(C) The Author(s) 2011. This article is published with open access at Springerlink.com

\begin{abstract}
Objective To asses the features and explore the clinical relevance of popliteal lymph nodes (PLNs) detected on MRI examination for different pathologies of the knee.

Materials and methods A total of 150 knee MRIs, which were conducted for various indications, were retrospectively collected from the Picture Archiving and Communication System. Imaging planes in at least two orthogonal planes were mandatory, with a field of view extending $15 \mathrm{~cm}$ cranial from the joint space. The localization of the PLN was determined by measuring the distance of the lowest border of the PLN to the lowest border of the lateral femoral condyle. Clinical diagnosis was obtained from radiology reports and a statistician performed the statistical analysis.

Results The patients were 70 males [mean age 36.6 years (range: 5-72 years)] and 80 females [mean age 41.1 years (range: $9-76$ years)]. In $36.7 \%$ of the patients, a PLN was
\end{abstract}

\section{G. D. Musters}

Master of Science at Academic Medical Center,

University of Amsterdam,

Amsterdam, The Netherlands

e-mail: Gijsbert.Musters@student.uva.nl

G. D. Musters $\cdot$ S. Bipat $\cdot$ M. Maas $(\bowtie)$

Department of Radiology, Academic Medical Center, University of Amsterdam,

Suite G1-211,

1105 AZ, Amsterdam, The Netherlands

e-mail: M.Maas@amc.uva.nl

S. Bipat

e-mail: S.Bipat@amc.uva.nl

\section{R. P. Kleipool}

Department of Anatomy, Embryology, and Physiology,

Academic Medical Center, University of Amsterdam,

Amsterdam, The Netherlands

e-mail: R.P.Kleipool@amc.uva.nl visible. The number of PLNs was negatively associated with age $(p<0.001)$. The mean number of PLNs was 0.5 PLN per patient. The mean length, height, and width were respectively: $0.57 \mathrm{~cm}(\mathrm{SD}=0.15), 0.84 \mathrm{~cm} \quad(\mathrm{SD}=0.26)$, and $0.71 \mathrm{~cm}(\mathrm{SD}=0.23)$. The mean location was $5.8 \mathrm{~cm}$ $(\mathrm{SD}=1.61)$. No association was found between the presence of PLNs and internal derangement, inflammation, or cancer $(p=0.368)$.

Conclusions PLNs appearance is age related, with a higher frequency at a young age. The presence of the PLNs showed no relation to a specific clinical situation.

Keywords Knee $\cdot$ Popliteal lymph node $\cdot$ Western population $\cdot$ Magnetic resonance imaging $\cdot$ Popliteal fossa

\section{Introduction}

In daily clinical practice, the presence of a popliteal lymph node (PLN) is not infrequently encountered when interpreting MRI examinations of the knee, especially when made for pediatric oncological indications [1]. If present, suspicion of metastatic disease has risen and further evaluation may be warranted. This is supported by the fact that clear cell sarcoma of ankle tendons and various dermatological oncological diagnoses may metastasize to PLNs $[2,3]$. The increased use of whole-body imaging in assessing the extent of oncological diseases might enhance this phenomenon [4], thus confronting radiologists with a difficult decision to make: should the encountered PLN be interpreted as dissemination of the oncology present or not?

Literature on this topic is scarce, especially in addressing the Western population. For this purpose, the following study was carried out. The aim of our research was to give a description of the prevalence of PLNs in a randomized 
Fig. 1 Representative images of the measurement of the dimensions and location. a Sagittal view of the knee. b Coronal view of the knee. Height: The height of a PLN. Length: The length of a PLN. Width: The width of a PLN. Location: Shortest distance between the lowest part of the PLN and lowest part of the lateral femur condyle
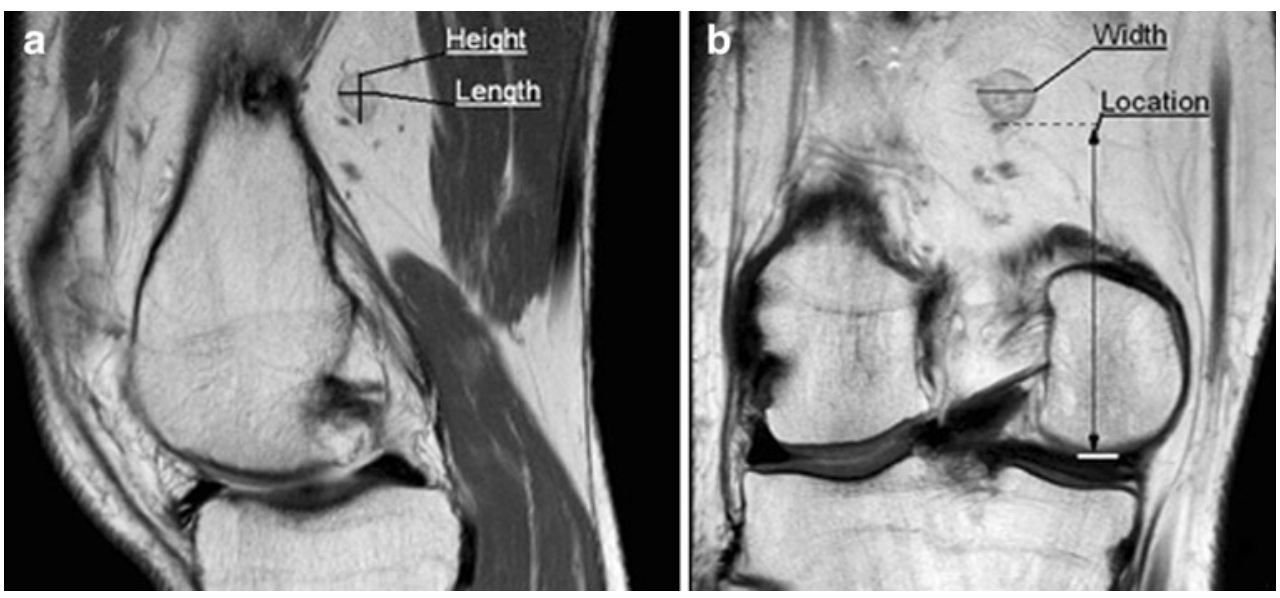

cohort of knee MRIs performed in our department, a radiology department in a tertiary referral center for pediatric and adult oncology, inflammatory diseases, and arthroscopic procedures. Specific features of PLNs, namely dimensions, location, and number, and the relation of PLN with patient age, sex, and specific clinical entities were analyzed.

\section{Materials and methods}

\section{Materials}

A retrospective cohort series of MRI examinations of the knee was collected from the Picture Archiving and
Communication System (PACS, from AGFA). The inclusion period was between October 2008 and December 2008, providing 199 consecutive MRI examinations. MRI examinations were performed on 1-1.5 T scanners (Siemens or Philips), using a dedicated knee coil. The following sequences were obtained: Proton-density (PD), short T1 inversion recovery (STIR), T1, T1 contrast, T1 contrast and fat saturation, $\mathrm{T} 2$, and $\mathrm{T} 2$ fat saturation.

\section{Methods}

Inclusion criteria were an imaging field of view assessing the entire knee extending cranially $15 \mathrm{~cm}$ to the lateral joint space/ condyle. PLNs were included if observed in at least two
Fig. 2 Association between age (years) and PLNs on MRI examination

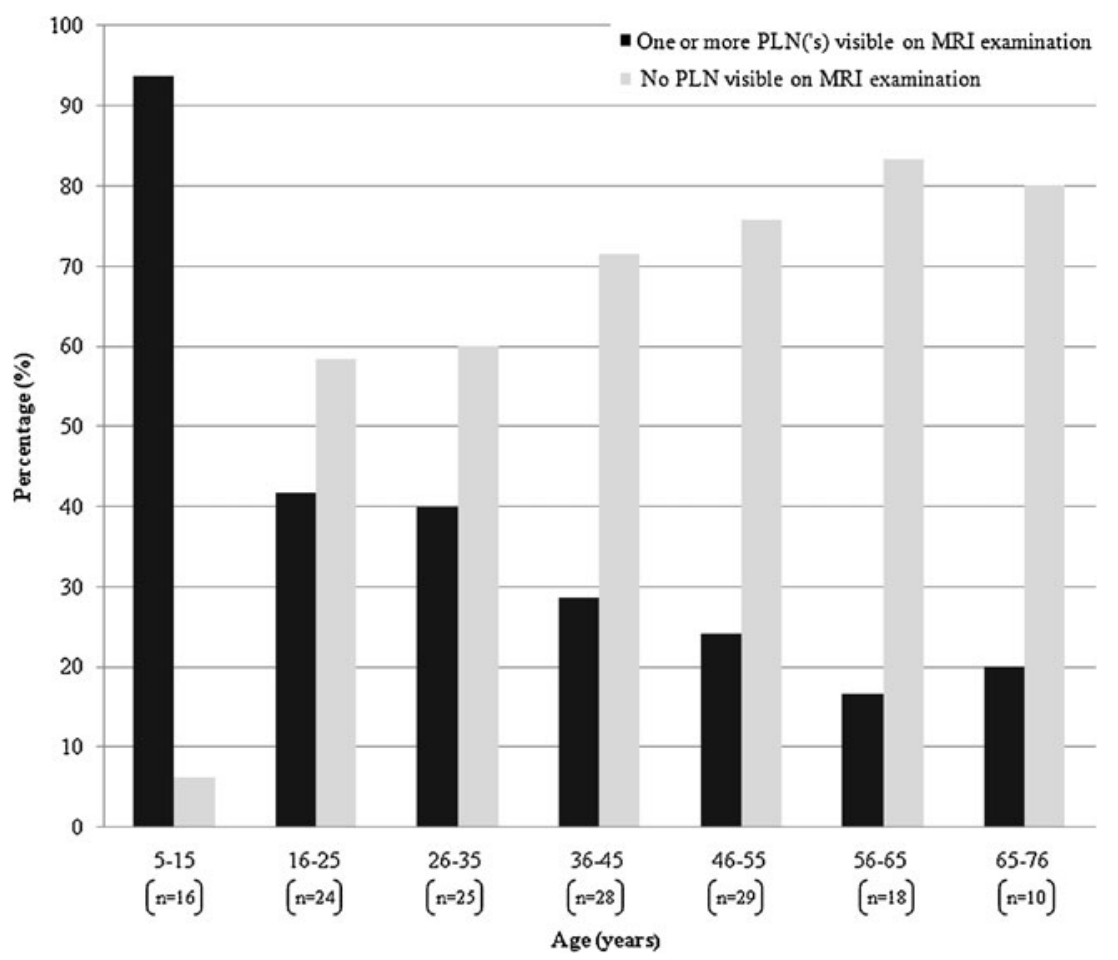


Table 1 The different clinical diagnoses groups of the 150 patients that were included

\begin{tabular}{lll}
\hline Clinical diagnoses of the MRI examinations & $n$ & $\%$ \\
\hline Internal derangement $^{\text {Inflammation }}{ }^{\mathrm{a}}$ & 71 & 47.3 \\
Cancer $^{\mathrm{b}}$ & 24 & 16.0 \\
Others $^{\mathrm{c}}$ & 8 & 5.3 \\
Total & 47 & 31.3 \\
& 150 & 100
\end{tabular}

${ }^{a}$ Inflammation included synovitis, rheumatoid arthritis, and juvenile idiopathic arthritis

${ }^{\mathrm{b}}$ Cancer included osteosarcomas, osteochondromas, giant cell tumor, atypical lipoma

${ }^{\mathrm{c}}$ Others included ganglion cyst, Baker's cyst, bone bruise, OsgoodSchlatter disease, no abnormality found, and muscle atrophy

imaging planes (axial, sagittal, or coronal). One observer (GDM) performed the evaluation of the MRI images.

All of the dimensions of the PLN were measured at their maximal dimensions and were determined as follows: the length of the PLN was measured on the sagittal or axial plane, the height was measured on the sagittal or coronal plane, and the width of the PLN was measured on the coronal or axial plane (Fig. 1). The location was measured as the shortest distance between the lowest border of the PLN and the lowest border of the lateral femur condyle. If more than one lymph node was detected, the average of the measurements of the PLNs was used, which was in concordance with the statistician. PLN identification was performed according to the description of a lymph node in the literature $[1,5]$.

\section{Statistical analysis}

Age was categorized into seven groups $(5-15,16-25$, $26-35,36-45,46-55,56-65$, and 66-76 years). The relationship between the presence of the PLNs and age was studied by Chi-square test for trend, as age was categorized in ordinal data. For the parameter dimensions, location, and number of PLNs means and confidence intervals were determined.

The relationship between the presence of PLN and disease status was studied by Chi-square test, as disease status was categorized in nominal data. Disease status was categorized in four groups: internal derangement, inflammation, cancer, and others. In addition, a pairwise comparison was preformed between the four groups by Chi-square test or Fisher's exact test.

A statistician (SB) performed statistical analysis using SPSS 16.0.1 (SPSS, Chicago, IL, USA). The medical ethical board declared that approval was not necessary, due to the retrospective nature of this diagnostic study.

\section{Results}

Of the 199 patients, 49 did not fulfill inclusion criteria. The 49 patients were excluded due to incomplete imaging planes. Of the remaining 150 patients, 70 were male [mean age 36.6 years (range: 5-72 years)] and 80 were female [mean age 41.1 years (range: 9-76 years)]. The age-related distribution was as follows: $5-15$ years $(n=16), 16-25$ years $(n=24), 26-35$ years $(n=25), 36-45$ years $(n=28), 46-$ 55 years $(n=29), 56-65$ years $(n=18)$, and 66-76 years $(n=$ $10)$. Of the included patients, the following sequences were obtained: PD $(n=137)$, STIR $(n=112), \mathrm{T} 1(n=45), \mathrm{T} 1$ fat saturation $(n=3), \mathrm{T} 1$ contrast $(n=11), \mathrm{T} 2(n=95)$, and T2 fat saturation $(n=16)$.

Of the 150 patients that were included, 55 (36.7\%) patients presented with a PLN on MRI examination. The PLNs were found in all age groups. The PLNs found on MRI examination were negatively associated with age $(p<0.001)$. The PLNs declined from $93.8 \%$ in the age group $5-15$ years to $20.0 \%$ in the age group 66-76 years (Fig. 2). The different clinical diagnoses groups of the 150 patients is shown in Table 1 and the mean number of PLNs per age group is described in Table 2.

Dimensions and location were both normally distributed. The mean length of the PLNs in the 55 patients with a PLN on MRI examination was $0.57 \mathrm{~cm}(\mathrm{SD}=0.15)$ with a range of $0.3-0.9 \mathrm{~cm}$. Other dimensions were the mean height, which was $0.84 \mathrm{~cm}(\mathrm{SD}=0.26)$ with a range of $0.4-1.5 \mathrm{~cm}$, and a mean width of $0.71 \mathrm{~cm}(\mathrm{SD}=0.23)$ with a range of $0.3-0.9 \mathrm{~cm}$. The mean location of the 55 patients with a PLN was $5.8 \mathrm{~cm}(\mathrm{SD}=1.61)$ cranial from the lowest border of the lateral femur condyle with a range of $1.8-12 \mathrm{~cm}$.

The most common clinical diagnosis of the 55 patients with PLNs was internal derangement (43.6\%). Internal derangement consisted of meniscal disruption, collateral ligament disruption, cruciate ligament disruption, fracture, and osteochondral defects. Inflammation was the clinical diagnosis in $21.8 \%$, and cancer in $7.3 \%$. Figure 3 describes the presence of the PLN on MRI examination for different

Table 2 Amount of PLN in different age groups

\begin{tabular}{llll}
\hline Age (years) & $n$ & Mean number of PLN & SD \\
\hline $5-15$ & 15 & 1.53 & 0.92 \\
$16-25$ & 10 & 1.30 & 0.68 \\
$26-35$ & 10 & 1.50 & 0.85 \\
$36-45$ & 8 & 1.25 & 0.46 \\
$46-55$ & 7 & 1.57 & 1.13 \\
$56-65$ & 3 & 1.00 & 0.00 \\
$66-76$ & 2 & 2.50 & 2.12 \\
Total & 55 & 1.45 & 0.86 \\
\hline
\end{tabular}


Fig. 3 The percentage of PLNs found in the different clinical diagnosis groups (for included different clinical diagnoses included in these groups see Table 1)

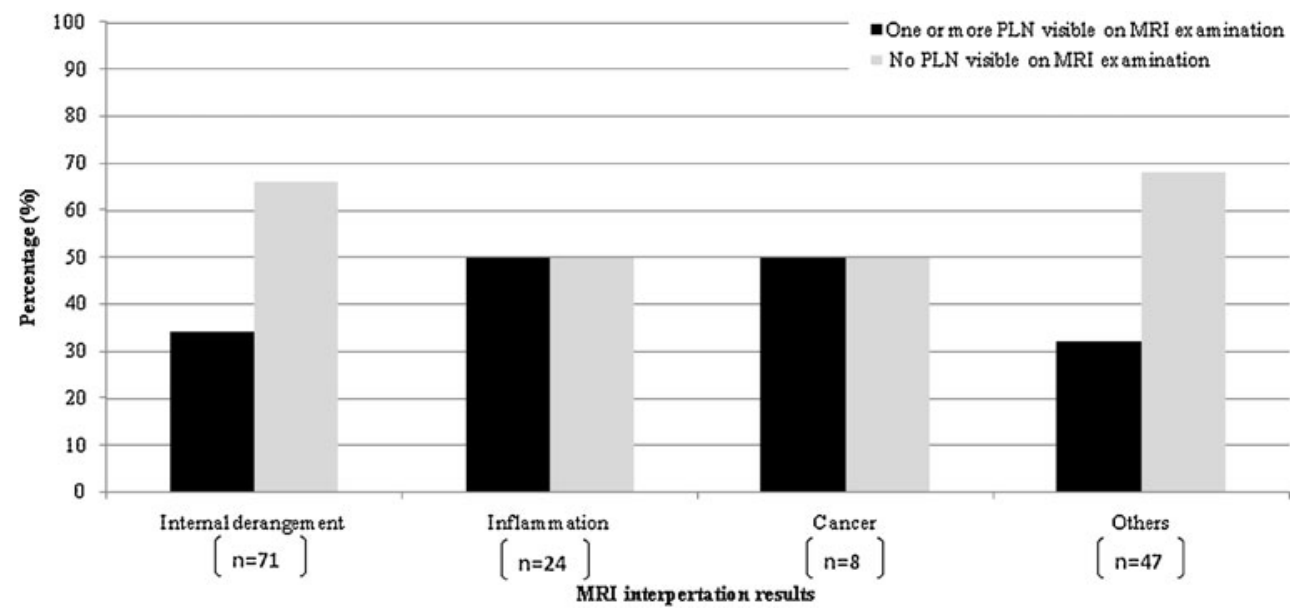

clinical diagnoses. An association between the various pathologies was not clinically significant $(p=0.368)$. For the included patients, the distribution of the various pathologies and age is described in Table 3.

\section{Discussion}

This study shows, to our knowledge for the first time, a rather high (36.7\%), inversely age-related frequency of the presence of PLNs, as seen on standard knee MRI examinations in a Western population. Nodes did not show significantly more frequent presence in oncology or inflammatory diseases.

Due to the limited literature, the significance of the presence of the PLN on MRI examination is generally not reported. However, which actions are advised in a given clinical situation with a patient with an oncological disease in the lower extremity? Currently, the presence of the PLNs on MRI examination is only evaluated when suspicion has arisen for metastasis from clear cell sarcoma of the ankle tendons and various dermatological malignancies [2, 3]. This study has generated the features of "normal" PLN distribution in a
Western population. When the encountered PLN differs from these data, one can consider this a pathological entity and biopsy might be warranted. One might expect this to become a more significant problem with the increased use of oncological whole-body imaging, in which STIR sequence will depict PLN that is non-fatty quite easily.

When compared to the sparse literature, mainly from South Korea, dimension, size, number, and inverse relation with age were similar, yet prevalence was lower in our Western cohort that was studied. The PLNs are reported to be largest in neonates and become smaller with age [1]. Due to the low antigenic stimulation, the PLNs undergo replacement of lymphatic parenchyma to fatty tissue (fatty change) [6]. Because the poplitea fossa has great fat signal intensity, the PLN becomes more difficult to differentiate from its surroundings, which affects its visibility on MRI examination. Besides fatty change, the PLNs can in some patients be too small to be visible on the MRI examination, so not every PLN is visible [1]. The number of PLNs is still lower than described in textbooks [7].

We need to consider the limitations of this study. The retrospective review of data in a small, yet non-selected cohort
Table 3 The various pathologies in all age groups

${ }^{\text {a }}$ Inflammation included synovitis, rheumatoid arthritis, and juvenile idiopathic arthritis

${ }^{\mathrm{b}}$ Cancer included osteosarcomas, osteochondromas, giant cell tumor, atypical lipoma

${ }^{\mathrm{c}}$ Others included ganglion cyst, Baker's cyst, bone bruise, OsgoodSchlatter disease, no abnormality found, and muscle atrophy

\begin{tabular}{|c|c|c|c|c|c|c|}
\hline & & \multicolumn{5}{|c|}{ Clinical diagnoses of the MRI examinations } \\
\hline & & $\begin{array}{l}\text { Internal derangement } \\
(n)\end{array}$ & $\begin{array}{l}\text { Inflammation }^{\mathrm{a}} \\
(n)\end{array}$ & $\begin{array}{l}\text { Cancer }^{\mathrm{b}} \\
(n)\end{array}$ & $\begin{array}{l}\text { Others }{ }^{\mathrm{c}} \\
(n)\end{array}$ & $\begin{array}{l}\text { Total } \\
(n)\end{array}$ \\
\hline \multirow[t]{8}{*}{ Age (years) } & $5-15$ & 2 & 8 & 1 & 5 & 16 \\
\hline & $16-25$ & 15 & 2 & 1 & 6 & 24 \\
\hline & $26-35$ & 12 & 4 & 1 & 8 & 25 \\
\hline & $36-45$ & 11 & 2 & 0 & 15 & 28 \\
\hline & $46-55$ & 16 & 5 & 2 & 6 & 29 \\
\hline & $56-65$ & 10 & 2 & 1 & 5 & 18 \\
\hline & $66-76$ & 5 & 1 & 2 & 2 & 10 \\
\hline & Total & 71 & 24 & 8 & 47 & 150 \\
\hline
\end{tabular}


size of a tertiary referral center might generate a selection bias. Furthermore, the cohort sizes of the different pathology groups were quite small. This hampers to firmly state that differences in lymph node prevalence in our subgroups are predictive. Yet since these are the first data arisen from a Western population, the authors consider this an important observation without any statement concerning significant positive relation to pathology. The study shows a significant inverse correlation with patient age. In our opinion, the results of this study can serve as a starting point for assessing the prevalence of the PLNs in the Western population.

Source of funding The authors declare that they have no conflict of interest.

Open Access This article is distributed under the terms of the Creative Commons Attribution Noncommercial License which permits any noncommercial use, distribution, and reproduction in any medium, provided the original author(s) and source are credited.

\section{References}

1. Moon HJ, Suh JS, Lee SH. Magnetic resonance appearance of normal popliteal lymph nodes: location and relationship of number, fatty change, and size of the lymph nodes with aging. Korean $\mathrm{J}$ Radiol Soc. 2002;47:665-71.

2. Hatta $N$, Morita $R$, Yamada $M$, Takehara $K$, Ichiyanagi $K$, Yokoyama K. Implications of popliteal lymph nodes detected by sentinel lymph node biopsy. Dermatol Surg. 2005;31:32730 .

3. Nishida Y, Yamada Y, Tsukushi S, Shibata S, Ishiguro N. Sentinel lymph node biopsy reveals a positive popliteal node in clear cell sarcoma. Anticancer Res. 2005;25:4413-6.

4. Krohmer S, Sorge I, Krausse A, Kluge R, Bierbach U, Marwede D, et al. Whole-body MRI for primary evaluation of malignant disease in children. European J Radiol. 2010;74:256-61.

5. Lai G, Rockall AG. Lymph node imaging in gynecologic malignancy. Semin Ultrasound CT MR. 2010;31:363-76.

6. Luscieti P, Hubschmid T, Cottier H, Hess MW, Sobin LH. Human lymph node morphology as a function of age and site. J Clin Pathol. 1980;33:454-61.

7. Moore KL, Dalley AF. Clinically oriented anatomy. 5th ed. Baltimore: Lippincott Williams and Wilkins; 2006. 